\title{
An Adaptive Timer Handoff Algorithm Based on Handoff Hysteresis
}

\author{
Weihong Cai ${ }^{1}$, Junhua Liu ${ }^{1}$, Chaoyang Lei $^{1}$ and Xia Fang ${ }^{2}$ \\ ${ }^{1}$ Department of Mobile communication, Hunan Post and Telecommunication \\ Vocational College, Changsha 410015, China \\ ${ }^{2}$ College of Computer Science and Technology, Hunan University of Arts and \\ Science, Changde 415000, China \\ liujunhua2010@126.com
}

\begin{abstract}
This paper sets forth an adaptive timer handoff algorithm with respect to the shortcoming of fixed timer handoff algorithm. In the algorithm, the real-time handoff timer value $T$ is calculated based on the distance $d_{i}$ between current mobile station and original serving cell as well as three constant variables: rank, ram and p, thus changing the handoff waiting time in real time. The simulation results showed that: the handoff occurs only when the mobile station needs to move to a far position from the original serving cell in the fixed timer handoff algorithm and larger set timer value $T$, and the strength of signals received by the mobile station in the original serving cell is poor; however, in the adaptive timer handoff algorithm, the ping-pong effect can be eliminated, the average handoff times are reduced, the mean strength of signals at handoff is better, the handoff position concentrates rationally and the overall performance reaches to the optimal effect.
\end{abstract}

Keywords: adaptive; timer; horizontal handoff; handoff hysteresis

\section{Introduction}

Attentions must be given to both the handoff times and signal strength of serving cell at handoff in a good mobile station handoff algorithm. In the algorithm based on the strength of signal received by the mobile station in the serving cell without considering the handoff hysteresis and handoff timer, a ping-pong handoff between both cells appears easily in the mobile station so that the mobile network load increases greatly as the strengths of signals received by the mobile station from both cells are very approximate, when the mobile station enters to an overlapped coverage area between serving cell and neighbor cell. To relieve the ping-pong handoff influence, the present practice is to improve the handoff threshold, namely, the fixed timer handoff algorithm is used for the handoff hysteresis. So, the chance of ping-pong handoff in the mobile station is reduced greatly, however, the mobile station at handoff may move to a far position from the original serving cell. Therefore, the strength of signal received by the mobile station from the original serving cell at handoff is weak extremely, and even the handoff is failed, thus increasing the handoff rate of missed call. Thus, this paper brings forth a modified handoff algorithm, namely, the adaptive timer handoff algorithm, which can not only eliminate the ping-pong handoff, reduce the mean handoff times in the mobile station, but also the mean strength of signals received by the mobile station from the original serving cell is better and the handoff position is centralized more rationally in the mobile station. 


\section{Fixed Timer Handoff Algorithm}

\subsection{Fixed Timer Handoff Algorithm based on Fixed Hysteresis}

Fixed Timer Handoff algorithm (FTHO) can be used to reduce the ping-pong handoff effect of the mobile station at handoff. In the event that the handover timer is $\mathrm{T}$ in the serving cell, if the strength of signal received by the mobile station from the neighbor cell is stronger than the sum of signal strength and hysteresis value $h$ in the serving cell in the sampling time $(\mathrm{K}-\mathrm{T}+1)$ th time, the timer is started. If this condition is met after sampling by continuously $\mathrm{T}$ times, the handoff occurs at the Kth sampling time and the determinant handoff formula is showed as below.

$$
\bigcap_{i=0}^{T-1}\left[\mathrm{RSS}_{\text {neighbour-cell, }} \mathrm{n}+\mathrm{i}>\mathrm{RSS}_{\text {serving-cell, } \mathrm{n}+\mathrm{i}}+\mathrm{h}\right]
$$

The handoff schematic diagram is shown in Figure 1. The handoff of neighbor cells can occur and the mobile station can hand over from serving cell 1 to neighbor cell 2 when the determinant handoff formula 1 is right after sampling by continuously $\mathrm{T}$ times; in the event that the strength of signal sampled from cell 2 is not greater than that from cell 1 in any time from $(\mathrm{K}-\mathrm{T}+1)$ th to Kth sampling times, the timer will restart counting.

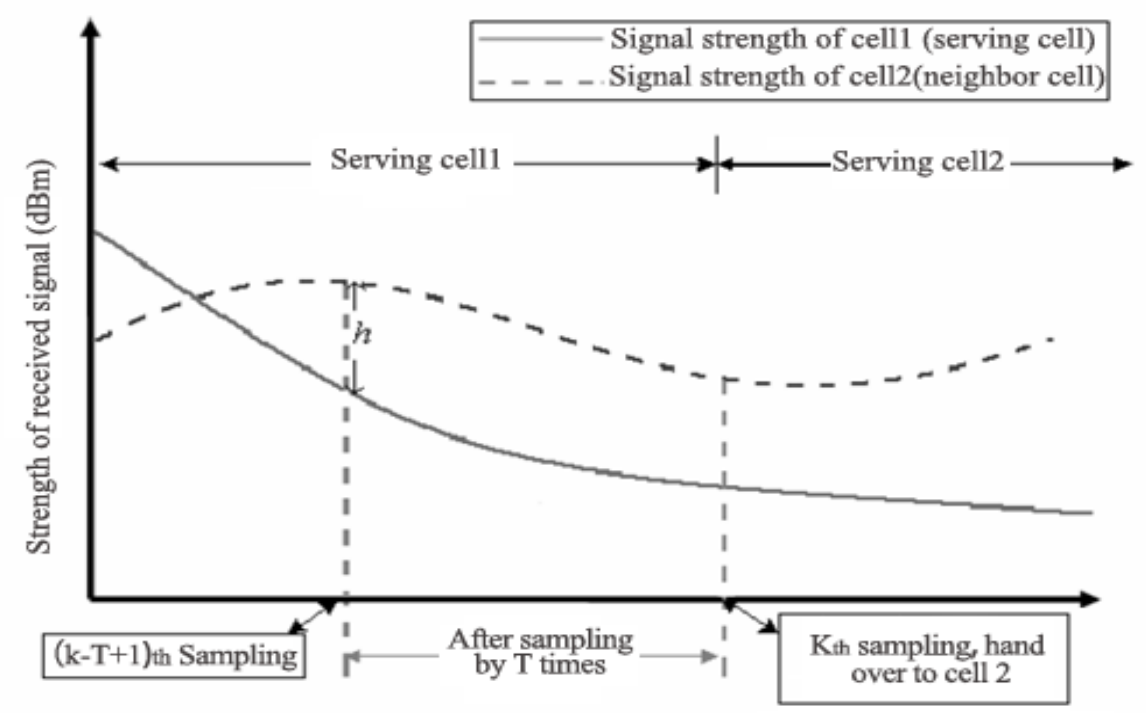

Figure 1. Schematic Diagram of Fixed Timer Handoff Algorithm

Apparently, if the timer value $\mathrm{T}$ is set larger, the handoff will be difficult. Although the handoff times and the ping-pong effect reduced, the mobile station moves to a far position from the serving cell 1 before the handoff may occur when the quality of connecting signal across the mobile station and serving cell is very poor.

\subsection{Fixed Timer Handoff Algorithm based on Adaptive Hysteresis}

In fixed hysteresis FTHO, the handoff hysteresis value is unchanged and the handoff quality of mobile station is poor as the factor of distance between the mobile stations and serving cell is not flexibly taken into account. So, the Adaptive Hysteresis Handoff algorithm 
(AHHO) was brought forward. In AHHO, the real-time hysteresis value is changed according to different distance between the mobile station and service cell. The real-time hysteresis value $\mathrm{H}$ is calculated in line with the formula 2.

$$
H=\max \left\{20 \times\left[-\left(\frac{d_{i}}{R}\right)^{4}\right\rceil, 0\right\}
$$

Where, $\mathrm{H}$ stands for the real-time hysteresis value, di stands for the distance measured between the mobile stations and serving cell in base station in the ith time and $\mathrm{R}$ stands for the radius of serving cell in base station. It is seen from formula 2 that the hysteresis value is larger and the handoff probability is lower when the mobile station is closer to the serving cell; on the contrary, the hysteresis value is less and the handoff probability is higher when the mobile station is further from the serving cell. Hence, the cell boundary is controlled essentially by the handoff position.

\section{Adaptive Timer Handoff Algorithm}

In comparison of AHHO and FTHO, the former can improve the ping-pong handoff effect of mobile station in both cell boundaries; however, it still has a higher handoff rate. When the mobile station locates in a junction of three cells, the strength of signals received by the mobile station is substantially approximate, the adaptive hysteresis value $\mathrm{H}$ is close to 0 when the ping-pong handoff effect cannot be solved. In addition, the possible longer fixed handoff time enables the signal received by the mobile station from the original serving cell at handoff is weak, and even the missed call occurs. Therefore, the disadvantages remain in FTHO. This paper proposes a new handoff algorithm, i.e., Adaptive Timer Handoff algorithm (ATHO).

The basic ATHO principle is described as below: the stronger the signal received by the mobile station from the original serving cell is, the larger the calculated handoff timer value $\mathrm{T}$ will be when the mobile station is nearer to the serving cell; on the contrary, the less the calculated timer value $\mathrm{T}$ is, the higher the handoff probability will be when the mobile station is further from the serving cell.

The determinant handoff formula in ATHO is equal to the formula 1; however, the timer value $\mathrm{T}$ varies with the distance between the mobile station and serving cell and its timer value $\mathrm{T}$ is calculated according to formula 3 .

$$
T=\operatorname{round}\left\{\max \left\{\operatorname{rank} \times\left(1-\left(e^{-\left(1-\frac{d_{i}}{r a m}\right)}\right)^{p}\right), 1\right\}\right.
$$

Where, the round represents a rounding function, max represents a function giving a maximum between two values inside a bracket, $d_{i}$, an exclusive variable in $T$ formula, represents the distance measured between the mobile station and the serving cell in the $i$ th time, rank, ram and $\mathrm{p}$ are constant variables, wherein rank is the maximum appropriate to the handoff timer value, ram is the minimum distance between the mobile station and the servicing cell when the handoff timer is changed to 1 , and $\mathrm{p}$ is the contrast index of distance. The influence of three constant variables on handoff timer value T is shown in Figure 2. 


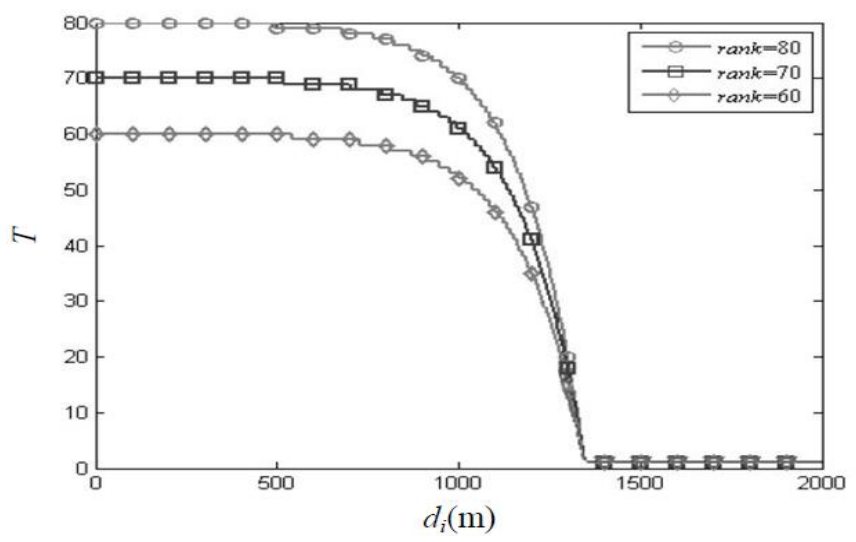

(a) At ram $=1350, \mathrm{p}=8$, influence of rank change on timer value $\mathrm{T}$

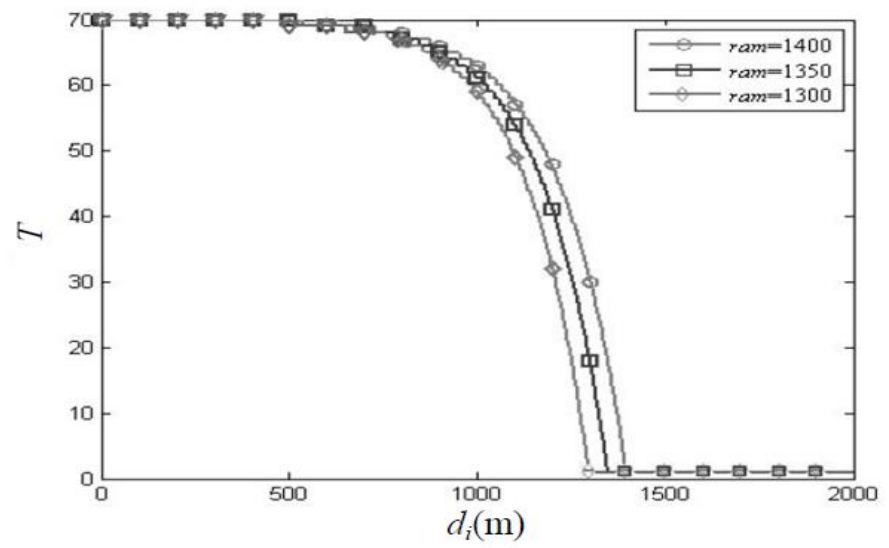

(b) At rank $=70, p=8$, influence of ram change on timer value $\mathrm{T}$

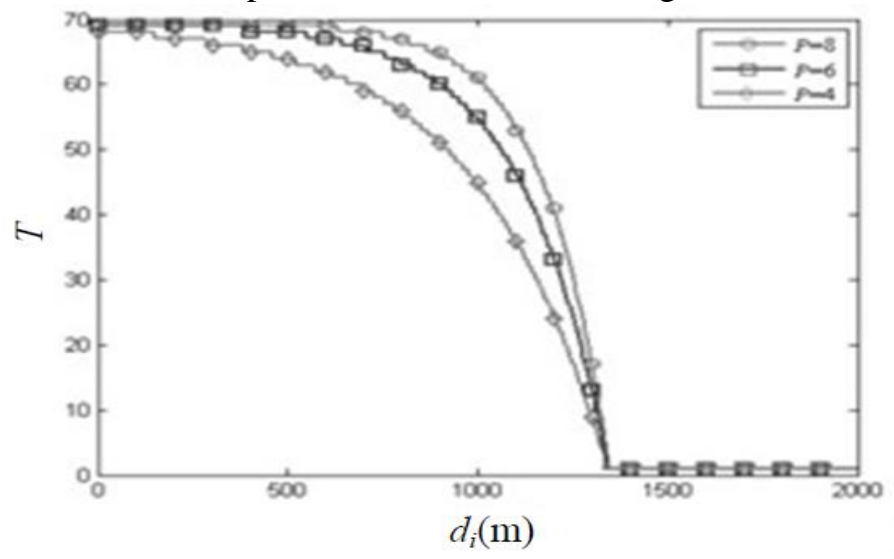

(c) At rank $=70$, ram $=1350$, the influence of $\mathrm{p}$ change on timer value $\mathrm{T}$

\section{Figure 2. Influence of Rank, Ram and p Changes on Timer Value T in Adaptive Timer Handoff}

Figure 2(a) shows that rank has larger influence on $\mathrm{T}$ in less $d_{i}$; the larger the rank is, the larger the timer value $\mathrm{T}$ will be; rank determines the max. $\mathrm{T}$ when $d_{i}$ is equal to 0 . With the increasing $d_{i}$, T will reduce regardless of rank value, the ultimate T will decline to 1 when $d_{i}$ is equal to ram. In the above Figure, as ram is set as 1350, $\mathrm{T}$ in three lines in above Figure 
declines to 1 when $d_{i}$ is equal to 1350. Figure 2(b) shows that T will decline with increasing regardless of ram value, and the ultimate $\mathrm{T}$ will decline to 1 when $d_{i}$ is equal to ram. Moreover, when $d_{i}$ is smaller, the influence of ram change on T is less; when $d_{i}$ is larger, the influence of ram change on $\mathrm{T}$ is larger. However, when $d_{i}$ is very large, the influence of ram change on $\mathrm{T}$ disappears. We also find that $\mathrm{T}$ will decline to 1 when ram and $d_{i}$ is larger. As shown in curve at ram $=1400$ in above figure, $\mathrm{T}$ will decline to 1 when $d_{i}$ is equal to 1400 ; and $\mathrm{T}$ will maintain as 1 when $d_{i}$ is larger than 1400 . Figure 2(c) shows that $\mathrm{P}$ value determines the vertical degree of the curve; the larger the $\mathrm{P}$ value is, the more vertical the curve will be. At ram=1350 in the above figure, T will decline to 1 when $d_{i}$ is equal to 1350 . Therefore, the under the change of three constant variables, rank, ram and $p$, the timer value $\mathrm{T}$ will increase with the declined $d_{i}$.

\section{Simulation Contrast of Different Handoff Algorithm Performance}

\subsection{Configuration of Simulation Condition}

Assuming that the mobile station starts from cell 1 each time and moves to cell 2 straightly in uniform speed, the mean handoff times, mean strength of signal received by the mobile station from cell 1 at handoff, and mean distance from cell 1 at first handoff were statistically analyzed through multiple simulation in computer in order to compare the performance of different horizontal handoff algorithms.

The formula for strength of signal received by the mobile station when moving from cell 1 to cell 2 is as below:

$$
\begin{aligned}
& \operatorname{RSS}_{\text {cell1 }}=\mathrm{K}_{1}-10 \times \mathrm{K}_{2} \times \log _{10}\left(\mathrm{~d}_{\mathrm{i}}\right)+£_{\mathrm{i}} \\
& \mathrm{RSS}_{\text {cell } 2}=\mathrm{K}_{1}-10 \times \mathrm{K}_{2} \times \log _{10}\left(\mathrm{D}-\mathrm{d}_{\mathrm{i}}\right)+£_{\mathrm{i}}
\end{aligned}
$$

Where, in formula 4 and $5, \mathrm{~K} 1$ indicates the base station transmission power, $\mathrm{K} 2$ indicates the path loss exponent, D indicates the distance between cell 1 and cell 2 , di indicates the distance of mobile station from cell 1 at the ith sampling time and £i indicates the influence of shade effect on signal strength of mobile station in the ith sampling time.

The specific simulation conditions are configured as in Table 1.

Table 1. Parameters of System Simulation

\begin{tabular}{|c|c|}
\hline the parameter name & parameter settings \\
\hline BS power & $\mathrm{K}_{1}=33 \mathrm{dBm}$ \\
\hline Velocity of mobile & $1 \mathrm{~meter} / \mathrm{sec}$ \\
\hline Sampling time & $1 \mathrm{sec}$ \\
\hline Correlation distance & 20 meter \\
\hline Path loss exponent & $\mathrm{K}_{2}=3$ \\
\hline Window size & $\mathrm{W}=4$ \\
\hline Shadow fading standard deviation & $\mathrm{S}=6 \mathrm{~dB}$ \\
\hline
\end{tabular}

\subsection{Influence of Rank, Ram and p Constant Variable Change on Handoff Position in ATHO}

Figure 3 shows the influence of rank, ram and $\mathrm{p}$ constant variables change on handoff position in ATHO. 
International Journal of Signal Processing, Image Processing and Pattern Recognition Vol.7, No.4 (2014)

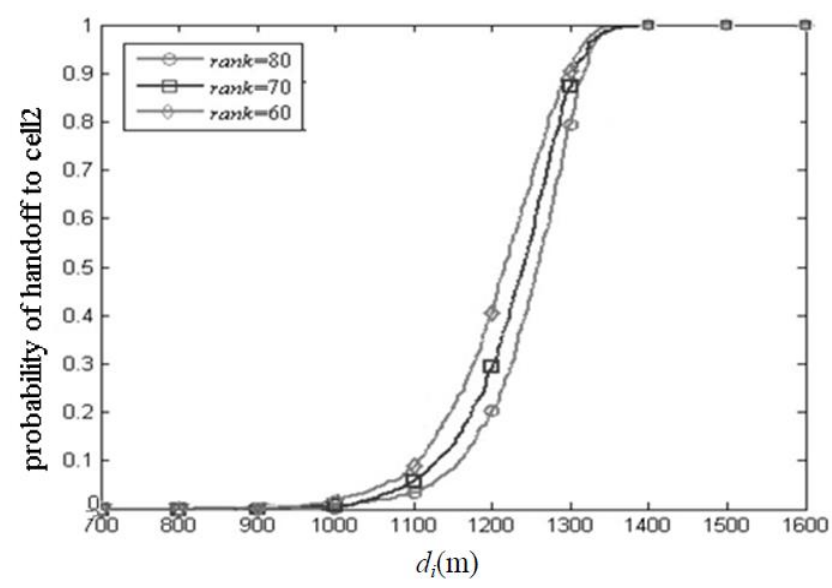

(a) At $\mathrm{p}=8$, ram $=1350$, influence of rank change on handoff position

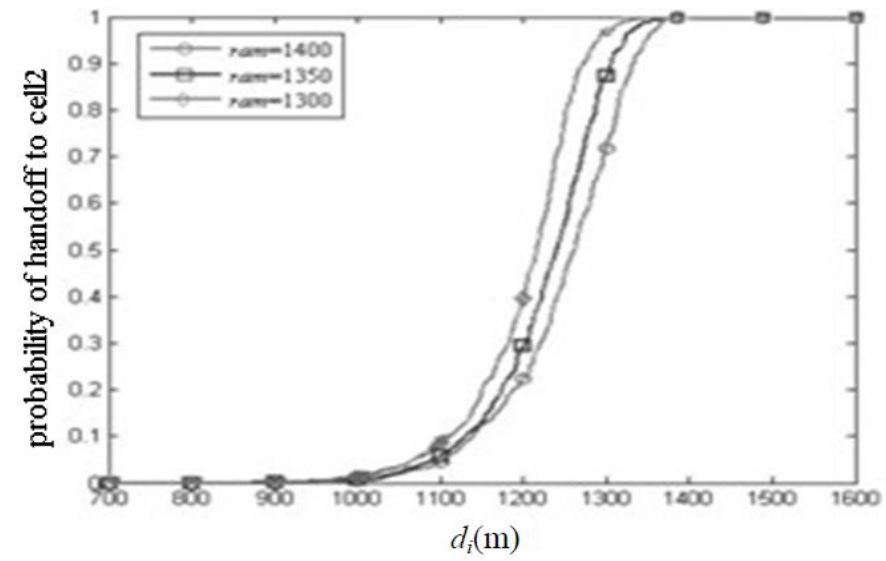

(b) At $\mathrm{p}=8$, rank $=70$, the influence of ram on handoff position

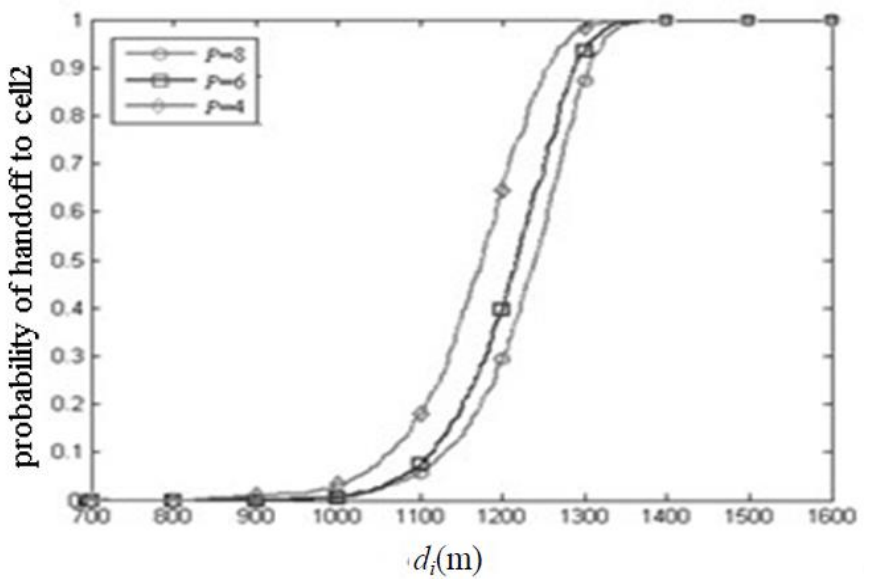

(c) At rank $=70, \mathrm{ram}=1350$, the influence of $\mathrm{p}$ on handoff position

\section{Figure 3. Cumulative Distribution Function about the Influence of Rank, Ram and P Constant Variable Change on Handoff Positions}

Figure 3(a) Figure 3(c), respectively, shows the cumulative distribution function about the influence of rank, ram and $\mathrm{P}$ changes on handoff positions. Figure 3(a) shows the 
cumulative probability distribution curve of handoff position is close to cell 2 with increasing rank, i.e., the handoff position is far away from cell 1 because the rank and $\mathrm{T}$ are larger when $d_{i}$ is less than or equal to ram. Figure 3(b) shows three curves are approximate because of less influence of ram on T when $d_{i}$ is less. However, with the increasing $d_{i}$, the influence of ram on $\mathrm{T}$ becomes apparent slowly. With larger ram, the cumulative probability distribution of handoff position is more approximate to cell 2 because the ram and T are larger. Figure 3(c) shows that the slope of cumulative probability distribution curves in handoff position rises when $\mathrm{P}$ increases, i.e., the curve is approximate to a vertical shape. Similarly, with larger P, the cumulative probability distribution in handoff position is approximate to cell 2 and the handoff is more centralized. However, the handoff of mobile station becomes less related to the strength of received signal. The determining basis of influence turns to $d_{i}$, and resultantly, the horizontal handoff based on the signal strength becomes the horizontal handoff based on position judgment.

\subsection{Contrast on Influence of FTHO and ATHO on Handoff Probability Density Distribution and Cumulative Handoff Probability Distribution}

In Figure 4 and Figure 5, the handoff probability density distribution and cumulative handoff probability distribution were compared at $\mathrm{T}=5, \mathrm{~T}=10$. The parameter $\mathrm{T}$ is appropriate to the below formula 6 in ATHO.

$$
T=\operatorname{round}\left\{\max \left\{70 \times\left(1-e^{\left.-\left(1-\frac{d_{i}}{1150}\right)^{8}\right)}, 1\right\}\right\}\right.
$$

Both figures show the observed handoff probabilities in 800 1400m distance as $d_{i}$ handoff probabilities are concentrated between $800 \sim 1400 \mathrm{~m}$. The hysteresis value H used for ATHO in above Figures is calculated with formula 1 to observe the probability distribution of different handoff algorithms.

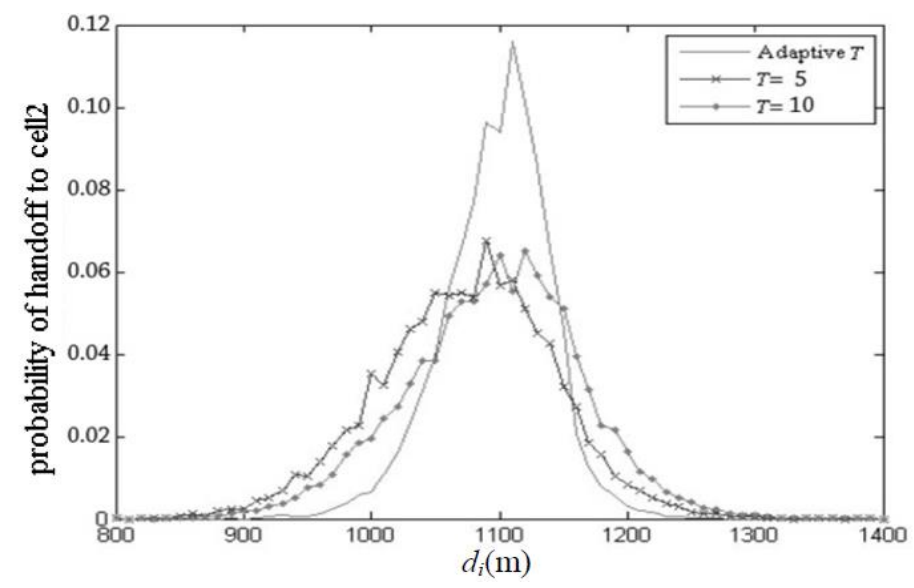

Figure 4. Contrast of Handoff Probability Density Distribution (PDF) 


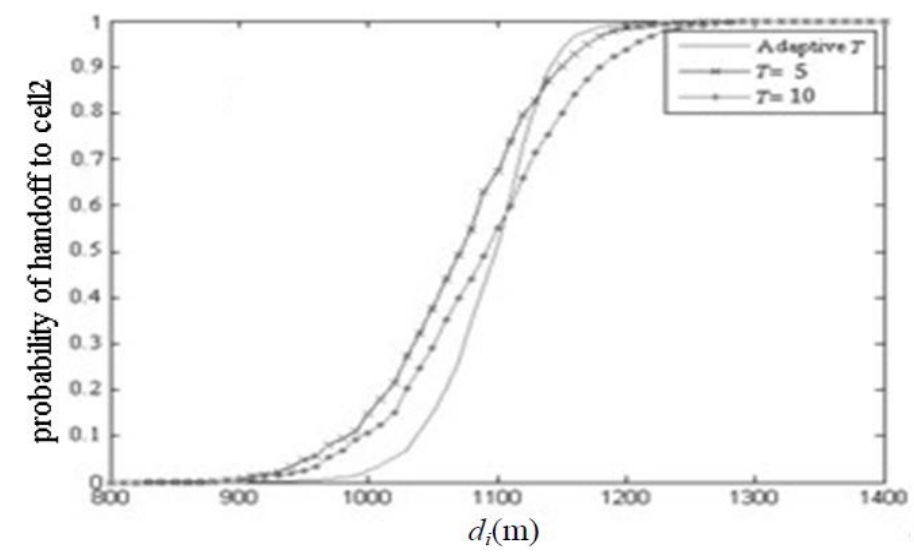

Figure 5. Contrast of Cumulative Probability Distribution (CDF)

Figure 4 and Figure 5 show that the mobile station hands over at about $1000 \mathrm{~m} 1150 \mathrm{~m}$ apart from the serving cell. When FTHO method is used at $\mathrm{T}=5$, the handoff probability in above Figures is prone to cell 1, namely, an early handoff occurs as the timer value is less. In early handoff, the strength of signals in serving cell at handoff is stronger; however, the pingpong handoff occurs easily. At $\mathrm{T}=10$, the handoff probability in above figures is prone to cell 2 , namely a later handoff occurs as the timer value is larger. The later handoff can reduce the ping-pong handoff times, but it also reduces the strength of signals in serving cell at handoff. Comparing to FTHO, the ATHO can control the handoff position within a centralized region in the range more effectively, namely a ping-pong effect is attained whilst considering the strength of signal in the original serving cells at handoff.

\subsection{Contrast of Average Handoff Times, Strength of Signal at Handoff and Handoff Position in Different Algorithms}

Table 2 and Table 3 demonstrate the simulation performance contrast of handoff times and strength of received signals at different hysteresis values in FTHO and ATHO algorithms. Apparently, in FTHO algorithm, when the hysteresis value is increased, the average handoff times are reduced whilst the strength of signal at handoff is reduced as well. Therefore, a trade-off will remain at choice of hysteresis value. By comparing the performances at $\mathrm{T}=5$ and $\mathrm{T}=10$ in FTHO algorithm, we might know that the strength of signal at handoff reduces though the average handoff times reduce at larger fixed timer value; contrarily, the strength of signal at handoff increases though the average handoff times increase at smaller fixed timer value. In ATHO algorithm, the handoff probability focuses on the junction of both cells or boundary of serving cell with the further distance between the mobile station and serving cell and reduced timer value. The data showed that both the average handoff times and strengths of signal at handoff in ATHO algorithm were better than FTHO at different T values.

Table 2. Contrast of Average Handoff Times

\begin{tabular}{|c|c|c|c|}
\hline & ATHO & FTHO $(\mathrm{T}=5)$ & FTHO $(\mathrm{T}=10)$ \\
\hline $\mathrm{H}=0 \mathrm{dBm}$ & 1.671 & 5.353 & 3.041 \\
\hline $\mathrm{H}=1 \mathrm{dBm}$ & 1.223 & 3.285 & 1.975 \\
\hline $\mathrm{H}=2 \mathrm{dBm}$ & 1.035 & 2.261 & 1.430 \\
\hline $\mathrm{H}=3 \mathrm{dBm}$ & 1.005 & 1.523 & 1.143 \\
\hline $\mathrm{H}=4 \mathrm{dBm}$ & 1.002 & 1.140 & 1.041 \\
\hline $\mathrm{H}=5 \mathrm{dBm}$ & 1.000 & 1.030 & 1.015 \\
\hline
\end{tabular}


Table 3. Contrast of Signal Strength $(\mathrm{dBm})$ at Handoff

\begin{tabular}{|c|c|c|c|}
\hline & ATHO & FTHO $(\mathrm{T}=5)$ & FTHO $(\mathrm{T}=10)$ \\
\hline $\mathrm{H}=0 \mathrm{dBm}$ & 1.671 & 5.353 & 3.041 \\
\hline $\mathrm{H}=1 \mathrm{dBm}$ & 1.223 & 3.285 & 1.975 \\
\hline $\mathrm{H}=2 \mathrm{dBm}$ & 1.035 & 2.261 & 1.430 \\
\hline $\mathrm{H}=3 \mathrm{dBm}$ & 1.005 & 1.523 & 1.143 \\
\hline $\mathrm{H}=4 \mathrm{dBm}$ & 1.002 & 1.140 & 1.041 \\
\hline $\mathrm{H}=5 \mathrm{dBm}$ & 1.000 & 1.030 & 1.015 \\
\hline
\end{tabular}

Table 4 shows the contrast of simulation performance at the first handoff position with different hysteresis values in ATHO and FTHO algorithms. From Table 4, we may find that the handoff distance is further from cell 1 when the hysteresis value is larger; the distance at handoff is further from cell 1 when $T$ is larger by comparing both fixed timer values. In observing FTHO conditions at $\mathrm{T}=5$, the average handoff position is more approximate to cell 1 than ATHO when the hysteresis value $\mathrm{H}$ is less than $4 \mathrm{dBm}$; the average handoff position is further from cell 1 than ATHO when the hysteresis value $\mathrm{H}$ is greater than or equal to $4 \mathrm{dBm}$; In FTHO at $\mathrm{T}=10$, the average handoff position is further from cell 1 than ATHO at handoff of mobile station when the hysteresis value $\mathrm{H}$ is greater than or equal to $1 \mathrm{dBm}$.

Table 4. Contrast of Handoff Position (m)

\begin{tabular}{|c|c|c|c|}
\hline & ATHO & $\begin{array}{c}\text { FTHO }(\mathrm{T}=5 \\
)\end{array}$ & FTHO $(\mathrm{T}=10)$ \\
\hline $\mathrm{H}=0 \mathrm{dBm}$ & 1.671 & 5.353 & 3.041 \\
\hline $\mathrm{H}=1 \mathrm{dBm}$ & 1.223 & 3.285 & 1.975 \\
\hline $\mathrm{H}=2 \mathrm{dBm}$ & 1.035 & 2.261 & 1.430 \\
\hline $\mathrm{H}=3 \mathrm{dBm}$ & 1.005 & 1.523 & 1.143 \\
\hline $\mathrm{H}=4 \mathrm{dBm}$ & 1.002 & 1.140 & 1.041 \\
\hline $\mathrm{H}=5 \mathrm{dBm}$ & 1.000 & 1.030 & 1.015 \\
\hline
\end{tabular}

Therefore, for FTHO algorithm, the handoff position is more susceptible to hysteresis value $\mathrm{H}$ and the handoff probability is distributed more broadly; for ATHO, the handoff probability is concentrated in the junction of both cells or boundary of cell 1 and the handoff positions is not susceptible to the hysteresis value. Just for the more concentrated probability of handoff position, the average handoff distance is often further; however, the stronger average signal strength may be gained at handoff.

Table 2 through Table 4 show the simulation results that: the shortcoming of poor strength of signal received in the serving cells thanks to FTHO algorithm can be improved when the handoff probability is concentrated in the junction of both cells or boundary of serving cell in ATHO algorithm.

Table 5 shows the contrast of handoff performance simulation with adaptive hysteresis under the absence of timer, presence of fixed timer and adaptive timer. 


\section{Table 5. Performance Contrast of Adaptive Hysteresis and Different Timer Handoff Algorithms}

\begin{tabular}{|c|c|c|c|}
\hline & $\begin{array}{l}\text { the average } \\
\text { number of } \\
\text { switching }\end{array}$ & $\begin{array}{l}\text { the average signal } \\
\text { strength when } \\
\text { switching }(\mathrm{dBm})\end{array}$ & $\begin{array}{l}\text { the average position } \\
\text { for the first Switch } \\
\text { time }(\mathrm{m})\end{array}$ \\
\hline Adaptive H(Average filter) & 1.823 & -94.676 & 961.3 \\
\hline Adaptive H (T=5) & 1.012 & -94.818 & 1117.3 \\
\hline Adaptive H (T=10) & 1.002 & -94.887 & 1161.1 \\
\hline Adaptive H (Adaptive T) & 1.001 & -94.011 & 1093.2 \\
\hline
\end{tabular}

As viewed from Table 5, the strength of signal merely using adaptive hysteresis combined with averaging filter is stronger than the fixed timer and the first handoff position is closer to cell 1 , nevertheless, the average handoff times are higher than the fixed timer handoff. This is because the handoff can occur only after multiple sampling judgments, the ping-pong effect as well as the handoff times are reduced in FTHO algorithm comparing to the ATHO algorithm that uses average filter without timer. However, the strength of signal in mobile station is lower at handoff in FTHO algorithm. Therefore, a trade-off must be made for the timer values when the FTHO algorithm is used. The simulation results also verified that the ATHO overcomes the shortcoming, not only eliminating the ping-pong effect, but also gaining a better signal strength at handoff.

\section{Conclusion}

This paper compared the handoff probability density distribution, cumulative handoff probability distribution, average handoff times, handoff positions, average signal strength at handoff in ATHO and FTHO; and concluded that ATHO has less average handoff times, more concentrated handoff positions and stronger signal strength at handoff than FTHO. Therefore, ATHO is better than FTHO in overall performance.

\section{Acknowledgment}

This work was supported by the National Science and Technology Major Special Projects of China (2010ZX03005-001-03,2012ZX03001-036-002), supported by the fund of Hunan Province Department of Education (12C0961), and supported by the Scientific Research Fund of Hunan Provincial Education Department, P.R.China (No.09C704).

\section{References}

[1] Y. F. Huang, C. W. Hsu, F. B. Gao and H. C. Chen, "Performance of Adaptive Vertical Handoff in Heterogeneous Networks of WLAN and WCDMA Systems", Proceedings of 5th International Conference on Networked Computing and Advanced Information Management (NCM2009), Seoul, (2009), pp. 2012-2017.

[2] M. Maitra, A. Mukherjee and D. Saha, "Integrated Inter switch Cable and Handoff Cost Minimizing Heuristics for Cell-to-Switch Assignment in Wireless Cellular Networks", IEEE Transactions on Vehicular Technology, vol. 58, no. 9, (2009) November, pp. 5074-5082.

[3] F. He, F. Wang and D. Hu, "Distance and Velocity Assisted Handoff Decision Algorithm in Heterogeneous Networks", Proceedings of Second International Conference on Future Generation Communication and Networking (FGCN 2008), Sanya, (2008), pp. 1509-1512.

[4] R. Tawil, G. Pujolle and O. Salazar, "A Vertical Handoff Decision Scheme in Heterogeneous Wireless Systems", Proceedings of 2008 IEEE Vehicular Technology Conference (VTC2008), Calgary, (2008), pp. 2626-2630.

[5] S. D. Roy, "A Timer based Handoff Algorithm for Multi-cellular Systems", Proceedings of 2008 Emerging Trends in Engineering and Technology (ICETET), Indian, (2008), pp. 819-822.

[6] T. Shu, M. Liu and Z. C. Li, "A Performance Evaluation Model for RSS-Based Vertical Handoff Algorithms", 
Proceedings of IEEE Symposium on 2009 Computers and Communications (ISCC 2009), Sousse, (2009), pp. 271-276.

[7] H. Inaltekin, S. B. Wicker, C. Mung and H. V. Poor, "On Unbounded Path-Loss Models: Effects of Singularity on Wireless Network Performance", IEEE Journal on Selected Areas in Communications, vol. 27, no. 7, (2009) September, pp. 1078-1092.

[8] A. Tibrewala, D. Pramanick, S. D. Roy and S. Kundu, "Signal Strength Ratio Based Handoff Algorithms for Cellular Networks", Proceedings of Annual IEEE 2008 India Conference (INDICON 2008), Indian, (2008), pp. 11-13.

\section{Authors}

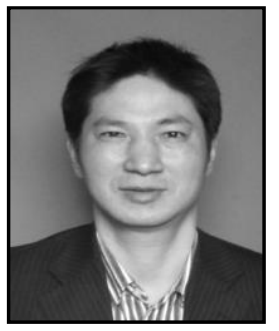

Weihong Cai, Male (1971), A master's degree, the Associate Professor of Mobile communication Department of Hunan Post and Telecommunication Vocational College, his research interests include mobile communication system, Mobile terminals.

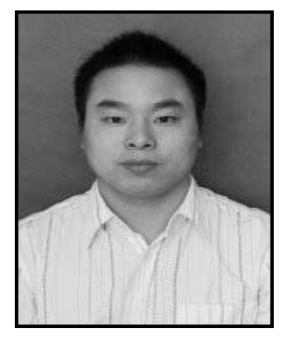

JunHua Liu, Male (1979), A master's degree, the lecturer of Mobile communication Department of Hunan Post and Telecommunication Vocational College. His research interests include the image processing, software engineering, mobile internet.

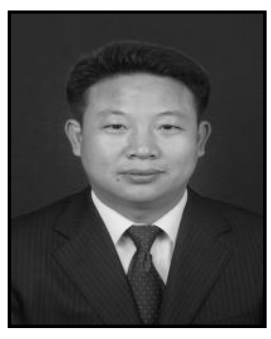

Chaoyang Lei, Male (1971), A master's degree, the Professor of Mobile communication Department of Hunan Post and Telecommunication Vocational College, his research interests include the image processing, computer network technology.

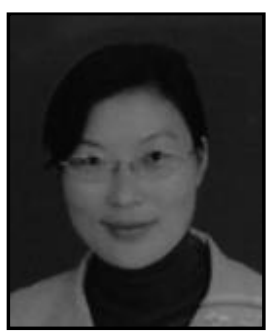

Xia Fang, Female (1972), A master's degree, the Associate Professor of College of Computer Science and Technology, Hunan University of Arts and Science, Her research interests include combinational Optimization and machine learning. 
International Journal of Signal Processing, Image Processing and Pattern Recognition Vol.7, No.4 (2014) 